\title{
Hoverboard: A Leap to the Future of Locomotion in VR!?
}

\author{
Jan Smeddinck ${ }^{1}$, Dmitry Alexandrovsky ${ }^{1}$, Dirk Wenig ${ }^{1}$, Michel Zimmer ${ }^{1}$, Waldemar \\ Wegele $^{1}$, Sylvia Juergens ${ }^{1}$, and Rainer Malaka ${ }^{1}$ \\ ${ }^{1}$ Digital Media Lab, TZI, University of Bremen, Germany \\ \{smeddinck, dimi, dwenig, ..., malaka\}@tzi.de
}

\begin{abstract}
Locomotion in virtual reality (VR) remains challenging due to limitations of common input methods. Sedentary input devices may endanger immersion, real-tovirtual world perception dissonance can lead to simulator sickness, and physical input devices such as framed walking dishes are often complex and expensive. We present a low-cost, easy to use, easy to manufacture, and easily portable device for locomotion in VR based on a hoverboard metaphor. Building on related work and our own iterative VR locomotion system designs we hypothesize that hoverboarding can provide a compelling and intuitive method for short- and long-distance locomotion in VR with a potential to reduce simulator sickness due to consistent and stable locomotion that corresponds well to the physical proprioception of the users while navigating VR. We discuss design iterations of our device prototypes, promising results from an early explorative evaluation, as well as ongoing continued work.
\end{abstract}

Keywords: Locomotion, Virtual Reality (VR), Motion-Based Control, Natural User Interface, Hoverboard, Leaning, Whole-Body Interface, Gaming.

\section{Introduction}

Virtual reality (VR) is expected to play an important role as a key new interaction modality for mainstream applications in entertainment computing, education, computer-supported cooperative work, and beyond. Due to the strong notion of physical presence and location in VR, applications require novel physical locomotion interfaces for navigation in virtual worlds which allow the user to travel long distances that cannot be bridged in reality while using the system due to physical constraints as well as due to constraints with tracking technologies.

In VR applications that present larger spaces and invite users to change locations, for example in many simulators or games, using traditional controllers, such as gamepads, or keyboard and mouse, is often cumbersome and unintuitive, since the input does not map well the impression of first-person movement that player-view camera translations in VR induce [1]. In many cases, a disconnect between the input method which leads to a specific sensory experience (including the vestibular system) for the user and the resulting displayed action can contribute to problems with VR sickness [2] and challenge immersion [3]. 
Modern VR systems offer "room-scale" tracking of physical motion (e.g., the HTC Vive), allowing the user to walk around in a small area. For traveling longer distances, there are approaches such as teleportation [4], walking-in-place (WIP) [5], or using walking dishes [6], treadmills [7], or spheres [8]. All of these approaches have specific advantages and disadvantages. They are either not fully immersive, or uncomfortable, or they require a complex or expensive hardware setup.

This motivated our iterative design exploration of further suspension devices following the original suspended walking system [5], targeting increased comfort, ease of use, lowered cost, immersion, reduced VR sickness, and facilitating faster and further locomotion. The resulting most favorable setup of a suspended wooden board (see Fig. 1, top) induced the emergence of the hoverboard metaphor and was integrated in a fully interactive VR locomotion prototype that was subjected to an early evaluation. The outcomes, while promising, indicated room for improvements, motivating iterations towards a followup modified hoverboard concept that is not suspended and targets increased portability, stability, and control.

This work contributes to the development of full-body interfaces for locomotion in VR with use cases in gaming and beyond. The publication of these latebreaking developments is meant to inform researchers and developers that may currently pursue similar challenges in the booming area of VR.

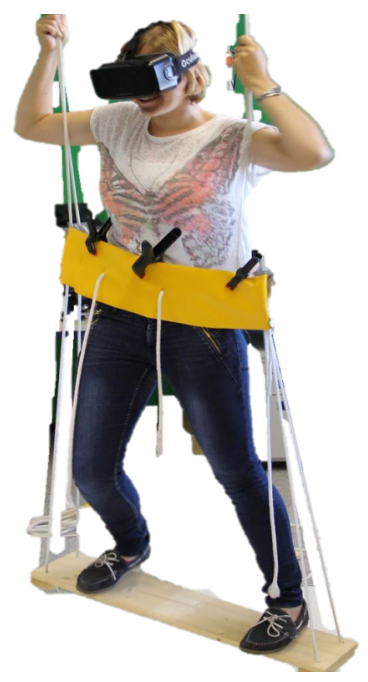

Fig. 1. Intermediate prototype from the iterative design process for a range of suspended setups for locomotion in VR.

\section{Related Work}

In the past, there have been commercial devices for body-based input that became popular for arcade of casual games. Instead of a VR setup the systems often realize visual output on a display, allowing users to reference the stationary environment around the display, thus motion sickness is not an issue. Sickness due to unconstrained movement in VR [3] is such a notable problem that most (even top-level) current VR games radically confine movement (e.g. Space Pirate Trainer) or use considerable workarounds, such as teleporting (e.g. Rec Room). Many acclaimed VR experiences (e.g. Elite Dangerous) are based on metaphors that match well with a seated computer desk setting and thus do not induce require physical locomotion. Some games use a "moving platform" (e.g. Hover Junkers), since standing on a surface that moves around the VR space matches better with actual proprioception if VR is used while standing, even if the floor is stable and does not move.

A considerable body of research explores the design space of traveling through virtual environments. Bowman et al. [3] provide a broad overview of techniques and devices. 
Stationary controls that are used in VR experiences with considerable locomotion mainly result in visual cues disregarding vestibular perception/feedback. This negatively affects a user's sense of presence and spatial awareness and also contributes to the risk of simulator sickness $[3,9]$. Involving a user's body in the interaction loop, providing multimodal feedback, can arguably help overcome these issues [1, 3, 9-12].

Hence, it is not surprising that a number of research projects have investigated interfaces that implement a walking metaphor. While many methods, such as walking in place [13], walking dishes and spheres [8], or treadmills [7] draw on walking motions that require users to actively carry their own weight, alternative suspended walking interfaces that utilize hanging harnesses have also been introduced [5]. Such walking-based methods offer an intuitive approach to locomotion in VR and can support immersive experiences [13, 14]. However, they suffer from shortcomings around latency, tracking accuracy, discomfort, restricted freedom of motion, or unnatural leg movements. Furthermore, they only work well with small virtual environments. Once a user is to explore large areas, techniques that involve walking, become exhausting [10]. Thus, other modalities, or mixed methods for traveling mark interesting venues for further exploration.

As one example, related work suggests the application of interfaces that rely on devices that are sensitive to weight shift, mapping a user's leaning motion to the direction and/or the speed of the device. Similar approaches have been discussed employing a "flying carpet" metaphor [3]. The Joyman [12] is an isotonic [3] VR locomotion interface that provides stability through resistive force that increases with displacement. A circular platform with a safeguard is connected via springs to a base. The inclination is measured with an inertia sensor. Along with the device the authors provide a translation model for the inclination of the device to a realistic control of virtual vehicles. Compared with a joystick, users completed navigation tasks significantly faster with the joystick, but had more fun and felt more present with Joyman. The interface encourages exaggerated leaning motions over the limit of balance. While this can be a fun way to navigate through some extreme sport games, such gestures appear inappropriate for regular navigation tasks. However, if the visual and auditory feedback presented in VR adequately corresponds to the movements that are enacted on the surface, such input schemes can provide close real-world-to-VR perceptive consistency.

Related work on vestibular feedback comparing a Wii Fit Balance Board as an isometric (i.e. non-displacing [3]) device with an isotonic device (the Reebok Core Trainer Tilt Board) found that, while no sig. diff. in performance between these two devices existed, participants preferred the isotonic interface, perceiving it as more joyful and realistic [1].

\section{Hoverboard System Design}

Based on related work and our own prior experience with suspension devices for locomotion in VR [5], an iterative design process was carried out to further investigate alternative suspended movement setups for locomotion in VR. The developments were motivated by the challenges around alternative techniques discussed above. Early informal evaluations with 
convenient subjects showed that the prototype devices pictured in Fig. 1 could support entertaining VR experiences, when test participants were asked to act out user generated gestures for direction control when using the setups while watching a VR video of moving down a rail that changed directions. In comparison, two alternative concepts of suspended walking and suspended flying were dismissed for reasons of discomfort and setup complexity when considering an interactive integration with a VR test application, and the third concept employing a hoverboard metaphor (Fig. 1) was chosen as first candidate for an interactive evaluation due to favorable ratings of comfort and ease of use.

\subsection{Hardware Design}

The hoverboard setup originally consisted of a wooden board attached by ropes to a roofmounted hold, allowing for rotation around the horizontal or vertical axis. A smartphone was attached to the bottom of the board as a readily available integrated sensor device with wireless network connectivity. Although this could easily be replaced by a dedicated embedded device for further developments, this setup allowed rapid iteration, benefitting from the integrated accelerometer, gyro, and compass for a high accuracy rotation detection [15]. A belt at waist level was added for safety reasons prior to testing the setup with users.

\subsection{Software Design}

A small android app employs the acceleration sensors and transfers the orientation data via Wi-Fi. For a simple, low-cost, and replicable approach, the Unity game engine was chosen to drive a VR test application. The design of the VR environment was deliberately kept simple (Fig. 2). It contains a virtual representation of the board that moves along hovering above a corridor or track for depth reference at a constant speed that was set to approximate $25 \mathrm{~km} / \mathrm{h}$. The level contains a series of crosses that are placed along the virtual track and can be collected by the user to induce steering movements and to measure precision. When a cross is collected, the counter in the HUD increases and the user hears a jingle sound. The application also plays a simple background music tune for ambience.

Incoming rotation values are interpolated to avoid jitter in movements and mapped to the rotation around vertical or horizontal axis of the virtual board according to the physical manipulation of the real wooden board. The application allows for a forward, as well as for a sideways stance orientation. The system was used in conjunction with an Oculus DK2.

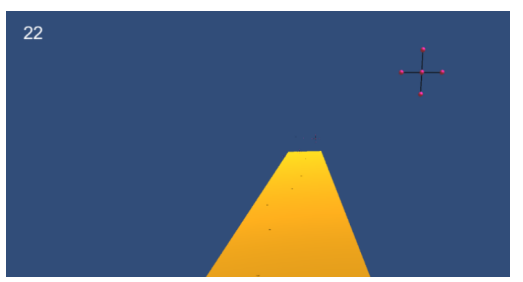

Fig. 2. Playful VR test application. Implemented in Unity3D.

\section{$4 \quad$ Evaluation}

An exploratory evaluation was carried out to capture first impressions regarding the general usability, user experience, and acceptance of the suspended locomotion method. It included four trials with different stance orientation (sideways vs. forward stance) and different perspectives (first-person vs. third-person), lasting about three minutes per trial. The users 
participated in an interview comparing the conditions and discussing the perception of the general approach regarding aspects of fun, precision of control, the level of immersion, the occurrence of motion-sickness, and a comparison to the applicability of traditional input methods (such as keyboard and mouse) for controlling similar locomotion in VR.

\subsection{Outcomes}

Four convenient subject casual gamer participants with an average age of 23 years were recruited for the study. None had used a VR headset before. However, one participant immediately reported VR sickness, which is not a rare occurrence when working with translational movement in VR. The participant did not complete the study and is thus not considered in the following results and analysis. None of the remaining participants reported sickness. Problems due to latency in tracking and smoothing were not observed. The remaining three subjects $(1 \mathrm{~m}, 2 \mathrm{f})$ all reported the interactions to be a fun experience. $\mathrm{P} 1$ and P3 explicitly related their impression to the control modality. Although no common preference could be isolated regarding the stance, the individual favorites appeared to correspond to the stance of their favorite stance-related sports. The third-person perspective was noted to provide a better impression of the orientation of the board. All felt immersed noted that they find the game boring without the hoverboard control scheme. P2 and P3 highlighted the role of whole-body input. P1 and P3 noted that they felt they had precise control of the board (P3 collected most crosses). P3 also commented on the performance and showed notable increases across trials. P1 and P2 attempted to collect crosses with imaginary virtual representations of their real (untracked) body parts (e.g. hands). P2 showed very pronounced movement, at one time shouting "this is great; I would immediately buy this".

\subsection{Discussion \& Limitations}

Overall, these exploratory evaluation results point towards a positive interaction experience. However, the evaluation is limited due to the low number of participants. Future evaluations should also consider using more immersive (real game) test applications, comparing to other control modalities, comparing the effect of controlling different objects that are not immediate representations of the input board, and controlling for the impact of first time VR experiences. In practical terms the most apparent limitation of the setup was a restricted portability, together with the need to provide hand holds due to fast rotations around the horizontal axis. This inspired the development of a follow-up prototype.
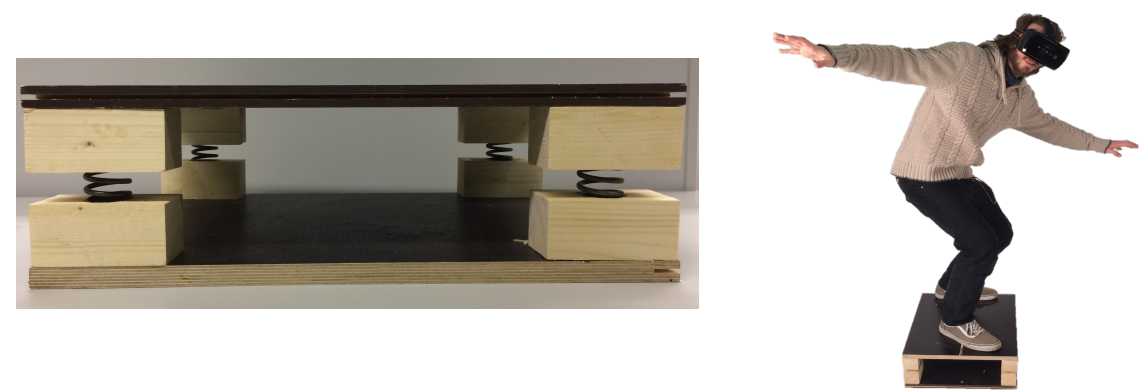

Fig. 3. The updated springboard iteration of the hoverboard device. 


\section{$5 \quad$ Updated Prototype}

Although the interaction method discussed above appeared promising, the setup is not easily portable. Thus, we developed a smaller device that utilizes an isotonic hoverboard interaction metaphor. It consists of two identical wooden boards that are connected by springs (Fig. 3). Again, a smartphone attached to the bottom side of the top board provides the inclination data to the host computer. For this prototype, the springs are chosen to bear a $70-80 \mathrm{~kg}$ person allowing an inclination up to $10^{\circ}$ in each direction. The springs can easily be swapped for different bodyweights and different maximum inclinations. One major advantage over the previous swing version is that it allows not only a leaning motion, but also to move freely on the board, which aims at facilitating improved tilting control.

\section{Discussion and Future Work}

The current prototype will be iterated to be fit for interactive demo use as well as to support further evaluations regarding the question of how the degrees of freedom can best be mapped to movement in VR, possibly combining velocity/speed and turning. Furthermore, a hydraulic suspension could support different weight ranges without requiring a manual swapping of the springs. We envision that the hoverboard concept can be used in entertainment computing applications as a simple and fun input device, tackling challenges of locomotion and travelling longer distances in virtual worlds. Hoverboard is as a simple, cheap, and easy to build device. As a physical input technique based on the user's body balance, it can be employed in fall prevention, for training core stability or a sense or space and time. It could also be used for spatial data exploration, e.g. traveling through detailed visualizations of the organs of a human body, or exploring large tags clouds or image collections, as well as to navigate geo-mapping environments such as Google Earth or StreetView. The hoverboard implements relative positioning (similar to a computer mouse or joystick) and, to allow for high immersion, it reacts to user input with dampened acceleration. This has advantages from the point of the user experience but presents problems regarding core usability metrics, such as time efficiency [12]. Therefore, we primarily envision application scenarios where efficiency is not a primary concern.

In many cases stationary controls are not well suited for immersive traveling in VR. Based on recent research we presented two iterations of full-body interfaces for locomotion in large virtual environments that are based on a hoverboard metaphor. First evaluation results promise a positive user experience and good control. Our latest prototype is a portable lowcost and easy to prepare device that promises to provide more precise control due to isotonic increasing resistance with increased stability, allowing for further explorations of the applicability of leaning for rotation and/or acceleration for long-range locomotion in VR.

\section{Acknowledgments}

This research is partially funded by the German Federal Ministry of Education and Research (BMBF). We thank the University of Bremen Spiele $A G$ for support with the latest prototype. 


\section{References}

1. Wang J., Lindeman R.W. (2012) Comparing Isometric and Elastic Surfboard Interfaces for Leaning-based Travel in 3D Virtual Environments. 3DUI 2012. IEEE, pp 31-38.

2. Klatzky R.L., Loomis J.M., Beall A.C., Chance S.S., Golledge R.G. (1998) Spatial Updating of Self-Position and Orientation During Real, Imagined, and Virtual Locomotion. Psychol. Sci. 9:293-298.

3. Bowman D.A., Kruijff E., Laviola J.J., Poupyrev I. (2004) 3D User Interfaces: Theory and Practice. Addison-Wesley, Boston.

4. Teleporting. In: HTC Vive Support. https://www.vive.com/us/support/category_howto/839430.html. Accessed 11 Jan 2017

5. Walther-Franks B., Wenig D., Smeddinck J., Malaka R. (2013) Suspended Walking: A Physical Locomotion Interface for Virtual Reality. ICEC 2013. Springer, pp 185-188.

6. Ohnishi K., Shibata M., Murakami T. (1996) Motion Control for Advanced Mechatronics. IEEE ASME Trans. Mechatron. 1:56-67.

7. Iwata H., Yano H., Nakaizumi F. (2001) Gait Master: A Versatile Locomotion Interface for Uneven Virtual Terrain. Proc. IEEE Virtual Real. 2001. pp 131-137.

8. Medina E., Fruland R., Weghorst S. (2008) Virtusphere: Walking in a Human Size VR "Hamster Ball.” Proc. Hum. Factors Ergon. Soc. Annu. Meet 52:2102-2106.

9. Ruddle R.A., Lessels S. (2006) For Efficient Navigational Search, Humans Require Full Physical Movement, but not a Rich Visual Scene. Psychol. Sci. 17:460-465.

10. Harris A., Nguyen K., Wilson P.T., Jackoski M., Williams B. (2014) Human Joystick: Wiileaning to Translate in Large Virtual Environments. VRCAI 2014. ACM, pp 231-234.

11. Kruijff E., Marquardt A., Trepkowski C., Lindeman R.W., Hinkenjann A., Maiero J., Riecke B.E. (2016) On Your Feet! Enhancing Self-Motion Perception in Leaning-Based Interfaces through Multisensory Stimuli. SUI 2016. ACM, pp 149-158.

12. Marchal M., Pettré J., Lécuyer A. (2011) Joyman: A Human-scale Joystick for Navigating in Virtual Worlds. 3DUI 2011. IEEE, pp 19-26.

13. Slater M., Usoh M., Steed A. (1995) Taking Steps: The Influence of a Walking Technique on Presence in Virtual Reality. ACM Trans. Comput.-Hum. Interact. (TOCHI) 2:201-219.

14. Williams B., Bailey S., Narasimham G., Li M., Bodenheimer B. (2011) Evaluation of Walking in Place on a Wii Balance Board to Explore a Virtual Environment. ACM Trans. Appl. Percept. 8:1-14.

15. Anjum A., Ilyas M.U. (2013) Activity Recognition using Smartphone Sensors. 10th Consum. Commun. Netw. Conf. IEEE. pp 914-919. 